\title{
The Role of Attitudes Toward
} Medication and Treatment Adherence in the Clinical Response to LAls: Findings From the STAR Network Depot Study

\section{OPEN ACCESS}

Edited by:

Norio Yasui-Furukori,

Dokkyo Medical University, Japan

Reviewed by:

Michele Fornaro,

New York State Psychiatric Institute

(NYSPI), United States

Roberto Cavallaro,

Vita-Salute San Raffaele

University, Italy

*Correspondence:

Andrea Aguglia

andrea.aguglia@unige.it

tThese authors share first authorship

$¥$ Members of the STAR Network Investigators group are provided in

the Acknowledgments

Specialty section: This article was submitted to Psychopharmacology, a section of the journal Frontiers in Psychiatry

Received: 27 September 2021 Accepted: 23 November 2021

Published: 16 December 2021

Citation:

Aguglia A, Fusar-Poli L, Amerio A, Placenti V, Concerto C, Martinotti $G$,

Carrà G, Bartoli F, D'Agostino A

Serafini G, Amore M, Aguglia E, Ostuzzi $G$, Barbui $C$ and the STAR

Network Investigators (2021) The Role of Attitudes Toward Medication and

Treatment Adherence in the Clinical Response to LA/s: Findings From the STAR Network Depot Study.

Front. Psychiatry 12:784366.

doi: 10.3389/fpsyt.2021.784366

\begin{abstract}
Andrea Aguglia ${ }^{1,2 * t}$, Laura Fusar-Poli ${ }^{3 \dagger}$, Andrea Amerio ${ }^{1,2}$, Valeria Placenti ${ }^{1,2}$, Carmen Concerto ${ }^{3}$, Giovanni Martinotti ${ }^{4}$, Giuseppe Carrà ${ }^{5,6}$, Francesco Bartoli ${ }^{5}$, Armando D'Agostino ${ }^{7}$, Gianluca Serafini ${ }^{1,2}$, Mario Amore ${ }^{1,2}$, Eugenio Aguglia ${ }^{3}$, Giovanni Ostuzzi ${ }^{8}$, Corrado Barbui ${ }^{8}$ and the STAR Network Investigators ${ }^{\neq}$
\end{abstract}

${ }^{1}$ Department of Neuroscience, Rehabilitation, Ophthalmology, Genetics, Maternal and Child Health, Section of Psychiatry, Istituto di Ricovero e Cura a Carattere Scientifico (IRCCS) Ospedale Policlinico San Martino, University of Genoa, Genoa, Italy, ${ }^{2}$ Istituto di Ricovero e Cura a Carattere Scientifico (IRCCS) Ospedale Policlinico San Martino, Genoa, Italy, ${ }^{3}$ Department of Clinical and Experimental Medicine, Psychiatry Unit, University of Catania, Catania, Italy, ${ }^{4}$ Department of Neuroscience, Imaging and Clinical Sciences, "G. d'Annunzio" University of Chieti, Chieti, Italy, ${ }^{5}$ Department of Medicine and Surgery, University of Milano-Bicocca, Monza, Italy, ${ }^{6}$ Division of Psychiatry, University College London, London, United Kingdom, ${ }^{7}$ Department of Health Sciences, Ospedale San Paolo, University of Milan, Milan, Italy, ${ }^{8}$ World Health Organization (WHO) Collaborating Centre for Research and Training in Mental Health and Service Evaluation, Department of Neuroscience, Biomedicine and Movement Sciences, Section of Psychiatry, University of Verona, Verona, Italy

Background: Long-acting injectable (LAl) antipsychotics are efficacious in managing psychotic symptoms in people affected by severe mental disorders, such as schizophrenia and bipolar disorder. The present study aimed to investigate whether attitude toward treatment and treatment adherence represent predictors of symptoms changes over time.

Methods: The STAR Network "Depot Study" was a naturalistic, multicenter, observational, prospective study that enrolled people initiating a LAl without restrictions on diagnosis, clinical severity or setting. Participants from 32 Italian centers were assessed at three time points: baseline, 6-month, and 12-month follow-up. Psychopathological symptoms, attitude toward medication and treatment adherence were measured using the Brief Psychiatric Rating Scale (BPRS), the Drug Attitude Inventory (DAl-10) and the Kemp's 7-point scale, respectively. Linear mixed-effects models were used to evaluate whether attitude toward medication and treatment adherence independently predicted symptoms changes over time. Analyses were conducted on the overall sample and then stratified according to the baseline severity (BPRS $<41$ or BPRS $\geq 41$ ).

Results: We included 461 participants of which 276 were males. The majority of participants had received a primary diagnosis of a schizophrenia spectrum disorder $(71.80 \%)$ and initiated a treatment with a second-generation LAl (69.63\%). BPRS, DAI-10, and Kemp's scale scores improved over time. Six linear regressions - conducted considering the outcome and predictors at baseline, 6-month, and 12-month follow-up independently-showed that both DAI-10 and Kemp's scale negatively associated 
with BPRS scores at the three considered time points. Linear mixed-effects models conducted on the overall sample did not show any significant association between attitude toward medication or treatment adherence and changes in psychiatric symptoms over time. However, after stratification according to baseline severity, we found that both DAI-10 and Kemp's scale negatively predicted changes in BPRS scores at 12month follow-up regardless of baseline severity. The association at 6-month follow-up was confirmed only in the group with moderate or severe symptoms at baseline.

Conclusion: Our findings corroborate the importance of improving the quality of relationship between clinicians and patients. Shared decision making and thorough discussions about benefits and side effects may improve the outcome in patients with severe mental disorders.

Keywords: long-acting injectable, antipsychotics, drug, attitude, adherence, therapeutic alliance, psychotic symptoms

\section{INTRODUCTION}

Severe mental illnesses are at higher risk of relapses associated with increased re-hospitalization rates, suicide attempts, behavioral abnormalities, persistent positive and residual symptoms, higher cognitive impairment, partial response with longer time to achieve remission, worsen quality of life and social functioning, and greater mortality and morbidity (1-5). A partial/absence adherence to pharmacological treatment is considered one of the most relevant risk factors for relapses (6), and it is influenced by several patient-, illness-, medication-, clinician-, and environmental-related factors (7-13).

It is well-known that oral and long-acting injectable (LAI) antipsychotics are similarly tolerable (14) and effective in the treatment of severe mental illnesses. Nevertheless, there is a literature debate on this topic, with randomized trials in clinically stable individuals showing a similar efficacy profile (15), and with large observational studies favoring LAI over oral antipsychotics in terms of re-hospitalizations (16-18). Observational studies might be less restrictive in terms of patient selection and have higher external validity, although an increased risk of detection, performance and prescribing bias may represent a relevant limitation (19). Although head-to-head comparison of LAIs did not show relevant differences between LAI treatments, aripiprazole, olanzapine, and paliperidone (1and 3-monthly formulation) might be considered first-line choices, as they are supported by the highest certainty of evidence for both relapse prevention and acceptability (20). Furthermore, benefits of LAI over oral formulation include improved bioavailability, maintenance stable plasma levels and receptor occupancy avoiding daily peaks, reducing plasma fluctuation, and increasing safety and tolerability, higher relapse prevention, greater compliance and earlier detection of nonadherence, requirement of regular visits with a psychiatrist specialist (21-23).

Given this evidence, guidelines did not provide any recommendations on which LAIs should be preferred but underlined the importance to reconsider these formulations for acute and long-term maintenance treatment rather than later stages of illness (24-26), even if the European rate of LAIs use in daily clinical practice is generally lower than $30-40 \%$ (27-32). Several barriers are recognized by clinicians, summarized as follows: needs of trained medical practitioners, poor in vitro-in vivo correlation, high cost, longer time to achieve maximum concentration, possibility of dose dumping, fear of injection, and perception of LAIs as painful and intrusive, difficult retrieval of drug in case of toxicity, increased variability among dosage unit, conceptualization that LAIs are only for chronic patients, perception of LAIs as punitive and coercive, subjective fear of not being able to control the dosage, self-stigma related to severe mental illness diagnosis and antipsychotics prescription (22, 32-35).

Recently, LAIs have been largely destigmatized by clinicians, and several strategies to increase LAIs use have been suggested $(35,36)$. They include drug-related interventions, such as simplified regimens and prevention of potential side effects to ameliorate adherence, patient-related interventions (i.e., psychosocial, behavioral, and personalized interventions), and service-related interventions (i.e., emerging technology-based monitoring, mobile applications, and artificial intelligence-based communications). In this context, a significant role is given by the so-called "shared decision-making," with a more patientinclusive approach, determining a balanced reasoned decision with a negotiation between clinician and patient $(37,38)$.

Leveraging data from a large observational, longitudinal, multicenter study involving individuals starting any LAI medication, the present study aimed to investigate whether attitudes toward treatment and adherence to therapy predicted changes in psychiatric symptomatology over time.

\section{MATERIALS AND METHODS}

\section{Sample}

This study was conducted according to STROBE (STrengthening the Reporting of OBservational studies in Epidemiology) 
statement items (39). The STROBE statement-checklist is provided in the Supplementary Table 1.

This is a large pragmatic, multicenter, observational, and longitudinal study, involving individuals starting any LAI medication. The detailed description of materials and methods of STAR Network Depot Study is provided on the first published paper (40). Another recently published paper considered followup data from the baseline recruitment phase (41).

The inclusion criteria for participants were: age $\geq 18$ years, starting a LAI medication, not taking any other LAI during the previous 3 months and willing to sign the written informed consent for voluntary participation. In particular, patients were consecutively recruited over a period of 12 months and assessed at 6- and 12-month follow-up. Individuals from any clinical setting, with any psychiatric diagnosis and any concomitant pharmacological treatment, were included in this study to trace the daily clinical activity in real-world. Participating centers are in- and out- patient services, including hospital psychiatric wards, mental health, and community day centers, residential facilities, part of the Italian STAR Network (Servizi Territoriali Associati per la Ricerca - Community Services Associated for Research), aiming to provide original data from real-world clinical practice $(42,43)$.

All participants signed a written informed consent prior their recruitment into the study, to have their clinical data potentially used for teaching and research purposes (provided that these data are anonymously treated). The study design was conducted in accordance with the guidelines provided in the current version of the Declaration of Helsinki (44). The protocol was approved by the local Ethics Committees of all participating centers and is publicly available at the Open Science Framework (OSF) online repository (https://osf.io/wt8kx/). The STAR Network Depot Study was not supported by any industry funding neither any monetary remuneration was provided for included subjects.

\section{Assessment}

A semi-structured interview used in previous published studies was administered to collect the socio-demographic characteristics, clinical, and pharmacological information (40, 41, 45, 46). Clinical interviews, health records and chart reviews were used to collect participants' baseline data while a follow-up form was used to collect psychopathological and pharmacological data.

In addition, specific psychometric tools were administered to investigate clinical dimensions, attitude toward medications and overall adherence to treatment, as follows: first, the clinicianrated Brief Psychiatric Rating Scale (BPRS) is validated in the Italian language by Roncone et al. (47) and assesses the overall level of psychiatric symptoms with the following severity: mild (31-40 total score), moderate (41-52 total score) and severe (above 52 total score) (48). This evaluation scale reported a high inter-rater reliability in both raters with high and low clinical experience (49). Second, the self-administered Drug Attitude Inventory 10 items (DAI-10) is validated in the Italian language (50) and assesses the patient attitude toward medications with a total score ranging from -10 to 10 . A higher total score indicates a better overall attitude toward medications with a positive attitude defined as a DAI-10 total score $>0$. Lastly, the clinician-rated Kemp's 7-point scale (51) is administered by the clinician and assesses overall adherence to treatment with a total score ranging from 1 to 7 . A higher total score indicates a better overall adherence to treatment with good acceptance defined as Kemp's total score $\geq 5$.

All clinicians participating in the recruitment and evaluation of patients performed a training meeting on the procedures and rating scales used in the study. These psychometric tools were administered at baseline and after 6 and 12 months of follow-up.

\section{Statistical Analysis}

Baseline and follow-up data were periodically forwarded from each recruiting center to the coordinating center (University of Verona), where they were entered into a computer database. Data correctness and consistency were ensured with the use of a double-entry technique and by applying a set of electronic and manual edit checks.

Continuous variables were expressed as means and standard deviations, whereas categorical variables were expressed as absolute numbers and percentages.

First, we conducted an ANOVA to evaluate the changes in the considered outcome (BPRS) and predictors (DAI-10, Kemp's 7point scale) scales over time. Second, six linear regression models - including the BPRS as outcome and the DAI-10 and the Kemp's scale as predictors - were computed independently using scores at baseline, 6-month, and 12-month follow-up (i.e., each association between the predictors and the outcome was calculated at the same timepoint). All regression models were adjusted for age and sex. Finally, linear mixed-effects models (LMEs) were used to analyze whether attitude toward medication (DAI-10) and adherence to treatment (Kemp's 7-point scale) were associated with changes in BPRS scores over time. LMEs were adjusted for age and sex (fixed effects), center and participant (random effects). LMEs were conducted on the overall sample and then stratified according to baseline severity. Specifically, the sample was divided into two groups (baseline BPRS $<41$ or $\geq 41$ ) based on the cut-offs proposed by Leucht et al. (48). The first group included patients with mild symptoms at baseline; the second group included patients with moderate or severe symptoms at baseline.

To reduce attrition bias, supplementary analyses using last observation carried forward (LOCF) approach were conducted by imputing the last observed scores on the collected variables (BPRS, DAI-10, and Kemp's scale). LOCF analyses were reported in Supplementary Tables 2, 3.

All statistical analyses and figures were carried out using Stata for Windows, version 16 (StataCorp, College Station, Texas, USA).

\section{RESULTS}

\section{Characteristics of Participants at Baseline}

At baseline, the sample comprised 461 participants recruited in 32 Italian centers, of which 276 were males. Mean age was $41.72 \pm 12.86$, with ages ranging between 18 and 76 years. Participants were mostly unemployed (49.24\%) and had never 
TABLE 1 | Characteristics of the sample $(N=461)$.

\begin{tabular}{|c|c|}
\hline Age, mean (SD) & $41.72(12.86)$ \\
\hline Sex, male, $N(\%)$ & $276(59.87)$ \\
\hline \multicolumn{2}{|l|}{ Education, $N(\%)$} \\
\hline Illiterate/no title & $7(1.52)$ \\
\hline Primary school & $28(6.07)$ \\
\hline Secondary school & $194(42.08)$ \\
\hline Diploma & $181(39.26)$ \\
\hline University & $45(9.76)$ \\
\hline Unknown & $6(1.30)$ \\
\hline \multicolumn{2}{|l|}{ Marital status, $N(\%)$} \\
\hline Never married & $324(70.28)$ \\
\hline Married & $67(14.53)$ \\
\hline Widowed & $4(0.87)$ \\
\hline Separated/divorced & $65(14.09)$ \\
\hline Unknown & $1(0.22)$ \\
\hline \multicolumn{2}{|l|}{ Employment, $\boldsymbol{N}(\%)$} \\
\hline Employed & $102(22.13)$ \\
\hline Unemployed & $227(49.24)$ \\
\hline Housewife & $24(5.21)$ \\
\hline Student & $16(3.47)$ \\
\hline Retired & $68(14.75)$ \\
\hline Other (e.g., sheltered employment) & $24(5.21)$ \\
\hline \multicolumn{2}{|l|}{ Primary diagnosis, $N(\%)$} \\
\hline Schizophrenia spectrum disorders & $331(71.80)$ \\
\hline Mood disorders & $84(18.22)$ \\
\hline Others & $46(9.98)$ \\
\hline \multicolumn{2}{|l|}{ Type of LAls, $\boldsymbol{N}(\%)$} \\
\hline First-generation LAl antipsychotics & $140(30.37)$ \\
\hline Second-generation LAl antipsychotics & $321(69.63)$ \\
\hline \multicolumn{2}{|l|}{ Concomitant oral medication, $N(\%)$} \\
\hline None & $38(8.24)$ \\
\hline 1 & $99(21.47)$ \\
\hline 2 & $156(33.84)$ \\
\hline 3 or more & $168(36.44)$ \\
\hline Substance abuse, $N(\%)$ & $91(19.74)$ \\
\hline Alcohol abuse, $N(\%)$ & 66 (14.32) \\
\hline
\end{tabular}

LAl, Long-acting injectable.

been married (70.28\%). The majority of participants received a primary diagnosis of a schizophrenia spectrum disorder $(71.80 \%)$ and initiated a treatment with a second-generation LAI (69.63\%). Socio-demographic and clinical characteristics of the sample are reported in Table $\mathbf{1}$.

\section{Changes of Psychiatric Symptoms (BPRS), Attitude Toward Drugs (DAl), and Adherence to Treatment (Kemp's 7-Point Scale) Over Time}

Multiple comparison ANOVA showed that psychiatric symptoms measured through the BPRS significantly improved over time $\left[F_{(2,686)}=259.98, p<0.001\right]$. Analogously, DAI-10 scores significantly increased, indicating a more confident attitude of participants toward medication $\left[F_{(2,678)}=22.78, p<0.001\right]$.
Finally, Kemp's scale significantly increased at 6- and 12 month follow-up, showing a patients' better adherence to pharmacological treatment $\left[F_{(2,686)}=20.02, p<0.001\right]$. Results are presented in Table 2.

\section{Association Between Attitudes Toward Medication, Treatment Adherence, and Symptoms Improvement at Baseline, 6- and 12-Month Follow-Up}

Linear regression analyses, adjusted for age and sex, showed a negative association between DAI-10 scores and BPRS scores at baseline $[B=-0.57(95 \% \mathrm{CI}-0.82,-0.32)], 6$-month $[B$ $=-0.63(95 \% \mathrm{CI}-0.85,-0.40)]$, and 12-month follow-up $[B=-0.65(95 \% \mathrm{CI}-0.88,-0.43)]$, indicating that a more positive attitude toward medication was associated with less severe psychiatric symptoms at each time point independently. Additionally, the Kemp's 7-point scale was negatively associated with BPRS scores at baseline $[B=-3.12(95 \% \mathrm{CI}-4.01,-2.22)]$, after 6 months $[B=-2.87(95 \% \mathrm{CI}-3.63,-2.11)]$ and after 12 months $[B=-2.97$ (95\% CI $-3.66,-2.28)]$ of treatment with LAIs. These results indicate that a higher compliance to therapy was related to less severe symptoms at each timepoint separately.

Figure 1 shows unadjusted linear prediction lines of BPRS on DAI-10 and Kemp's 7-point scale at baseline, 6-month, and 12-month follow-up.

\section{Association Between Attitudes Toward Medication, Treatment Adherence, and Change in Psychiatric Symptoms Over Time}

Considering the overall sample, LMEs adjusted for age and sex showed no significant effects between the DAI-10 and the Kemp's 7-point scale and changes in BPRS scores (Table 3). These results were confirmed by the LOCF analyses (Supplementary Table 2).

After stratifying the sample according to baseline severity, DAI-10 was significantly associated with changes in BPRS scores at 6-month follow-up in the group with baseline BPRS $\geq 41$ $[B=-0.32(95 \% \mathrm{CI}-0.61,-0.03)]$ and at 12 -month followup in both groups [BPRS $<41: B=-0.35$ (95\% CI -0.66 , $-0.05)$; BPRS $\geq 41: B=-0.41(95 \% \mathrm{CI}-0.71,-0.11)$ ]. Similarly, Kemp's 7-point scale was associated with changes in BPRS scores at 6-month evaluation in the group with the greatest severity at baseline $[B=-1.48(95 \% \mathrm{CI}-2.53,-0.43)]$, and after 12 months in both groups [BPRS $<41: B=-1.82(95 \% \mathrm{CI}-2.88,-0.77)$; BPRS $\geq 41: B=-1.46(95 \%$ CI $-2.51,-0.41)$ ] (Table 4).

LOCF analyses confirmed only the findings related to the group including patients with moderate and severe symptoms at baseline. Contrarywise, the group with the mildest symptoms did not show any significant association between DAI-10 or Kemp's and changes in BPRS score over time in LOCF analyses (Supplementary Table 3).

\section{DISCUSSION}

The present study investigated the relationship between attitude toward medications, treatment adherence, and changes in 
TABLE 2 | Repeated measures ANOVAs evaluating changes of the brief psychiatric rating scale (BPRS), drug attitude inventory (DAl-10), and Kemp's 7-point scale scores over time.

\begin{tabular}{|c|c|c|c|c|c|c|c|c|}
\hline & \multicolumn{2}{|c|}{ Baseline } & \multicolumn{2}{|c|}{6 months } & \multicolumn{2}{|c|}{12 months } & \multirow[b]{2}{*}{$\boldsymbol{F}$} & \multirow[b]{2}{*}{$p$} \\
\hline & $N$ & Mean (SD) & $N$ & Mean (SD) & $N$ & Mean (SD) & & \\
\hline BPRS & 461 & $48.96(14.67)$ & 357 & $36.50(11.12)$ & 331 & $35.48(11.06)$ & 259.98 & $<0.001$ \\
\hline DAl-10 & 459 & 2.00 (5.33) & 355 & 3.37 (5.05) & 326 & 3.92 (5.07) & 22.78 & $<0.001$ \\
\hline Kemp's scale & 460 & $4.79(1.44)$ & 357 & $5.25(1.48)$ & 332 & $5.33(1.56)$ & 20.02 & $<0.001$ \\
\hline
\end{tabular}

BPRS, Brief Psychiatric Rating Scale; DAI-10, Drug Attitude Inventory; SD, Standard deviation.

psychiatric symptoms in a large sample of ordinary practice patients that received treatment with LAIs over a period of 1 year.

First, our study showed improvement of attitude toward medication and treatment adherence over the course of the study, alongside with improvement of psychiatric symptoms. This is in line with recent meta-analyses, showing benefits of LAIs prescription in early psychosis (52), as well as in the treatment maintenance of schizophrenia (18, 20), not only in research settings but also in real-world clinical practice. Second, our findings support an association between the considered predictors and severity of psychiatric symptoms at the three selected timepoints, when considered independently. Nevertheless, examining this prediction longitudinally in the overall sample, we found that neither attitude toward medication nor treatment adherence was associated with changes in psychiatric symptomatology at 6- and 12-month follow-up.

The characteristics of the recruited sample may represent a reason of this non-significant finding. A large majority of the patients included in our study were affected by schizophrenia spectrum disorders, a group of severe chronic conditions frequently associated with poor insight levels (53). Previous studies have reported that insight represents one of the most important factors influencing attitude toward treatment, with higher levels of insight associated to a more positive attitude $(54,55)$. Poor illness insight has also been related to low adherence to treatment in psychotic patients, according to a recent systematic review (13). Another potential explanation is related to the combined use of LAIs and oral medication in this study. In fact, a large majority of participants $(91.8 \%)$ were taking concomitant oral psychotropic medications. If one hand LAIs are administered at regular intervals in outpatient services or at home under the supervision of a nurse and a psychiatrist, on the other hand the current compliance to oral medications was more difficult to control. Therefore, there might have been a discrepancy between the compliance to LAIs and to oral medications. Moreover, we cannot exclude that patient taking specific types of oral medication, such as supplementary antipsychotics or anticholinergics to alleviate extrapyramidal symptoms, may have had different responses on the individual attitude toward treatment or adherence to therapy. Indeed, side effects represent one of the most important reasons for LAI discontinuation in clinical practice (56). This notion has been confirmed also in this sample, as reported in our previous paper (41): reasons for discontinuation were mainly ascribed to the onset of adverse events (32.9\%), followed by participant refusal of the medication (20.6\%). Interestingly, discontinuers showed significantly lower DAI-10 and Kemp's 7-point scale than non-discontinuers.

From a methodological point of view, it is worth mentioning that while both the BPRS and the Kemp's 7-point scale are clinician-rated tool, the DAI-10 was completed by participants. Self-report tools might not be reliable in people affected by severe mental illnesses, particularly in psychotic patients (57). Thus, it is possible that attitude toward treatment was not adequately reported by patients included in our analysis. Moreover, more frequent follow-up visits (i.e., monthly) might have been useful to detect subtler changes, especially at the beginning of treatment with LAIs, when it is crucial to establish an optimal therapeutically alliance with patients who are initiating a new treatment. Nevertheless, given the naturalistic and pragmatic design of the study, the frequency of follow-up visits was widely variable across recruiting centers and could not be considered in our analysis.

It is interesting to note that, after stratifying the sample according to baseline severity, our results have changed. In fact, we found that improvement in both treatment adherence and attitude toward medication was associated with a significant reduction in BPRS scores after 12 months of treatment with LAI regardless of baseline severity. A significant negative association was also found after 6 months in the group with the greatest symptom severity at baseline. It is possible to hypothesize that the stratified analyses have minimized, at least in part, some of the inter-individual differences discussed above. Moreover, significant results appear more precocious in the group with moderate-to-severe symptoms at baseline. One possible reason is that the initiation of treatment with LAI in the group with the greatest severity may have led to a quicker stabilization of symptomatology and acquisition of illness insight, and thus to a better adherence to oral medication and to a more positive attitude toward treatment. On the contrary, the group already in remission or with mild symptoms (BPRS $<41$ at baseline) may have had only subtle changes in the first period of treatment with better improvements in the long-term. This is also confirmed by the LOCF analyses that showed no significant associations between predictors and symptoms changes over time in the group with BPRS $<41$ at baseline.

Overall, our findings lead to a critical reflection on the importance of the quality of relationship between clinicians and patients and the necessity to provide information regarding 


\section{BPRS/DAI-10}
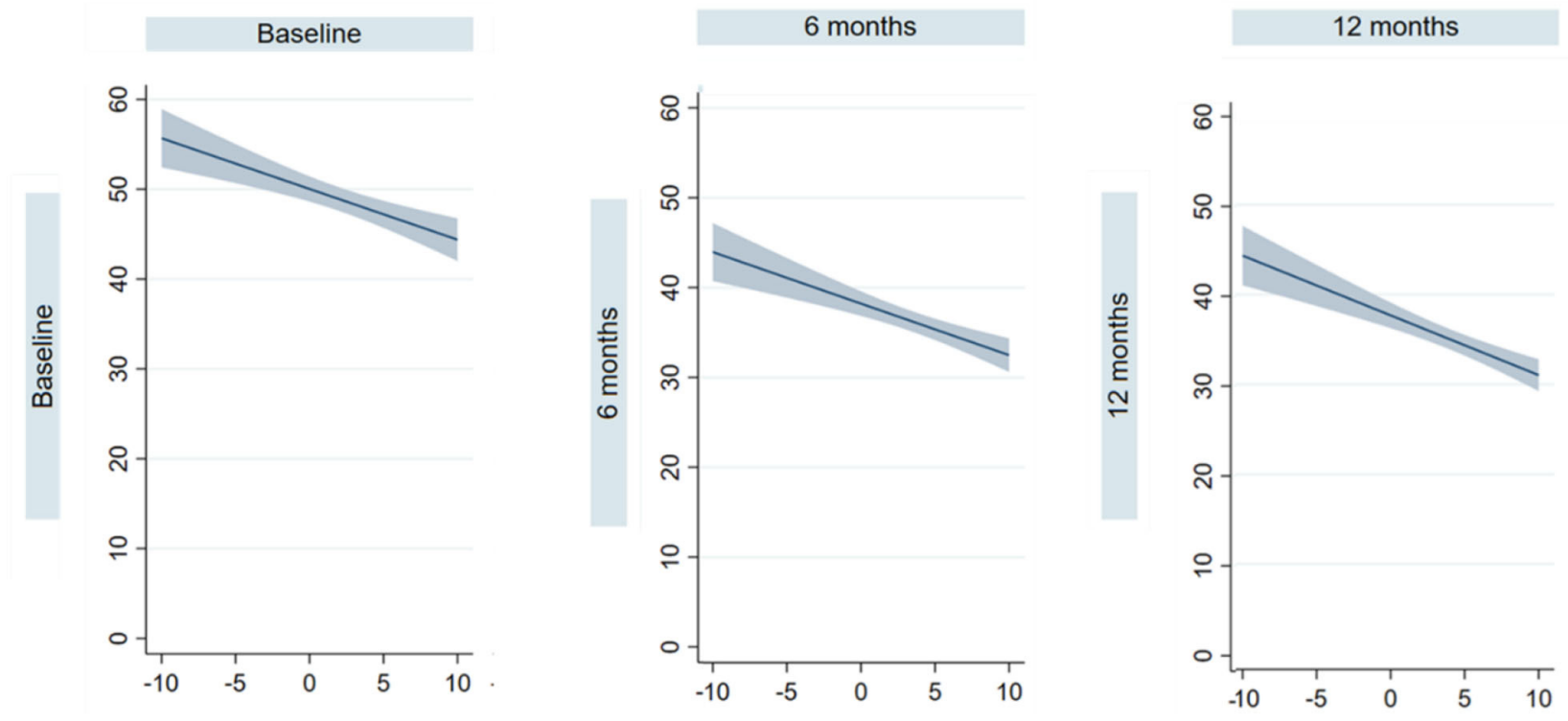

\section{BPRS/KEMP'S}
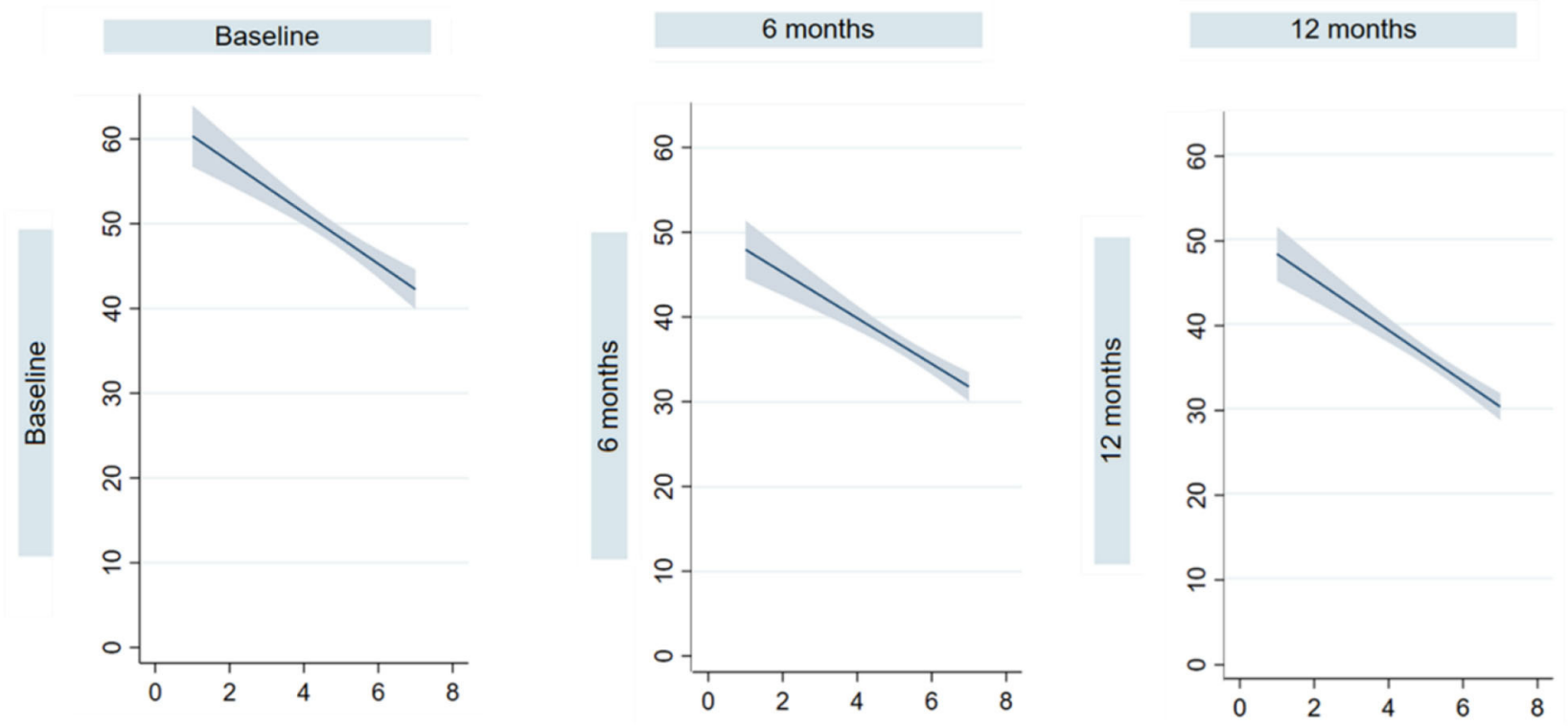

FIGURE 1 | Unadjusted linear prediction lines and 95\% confidence intervals (Cl) of the Brief Psychiatric Rating Scale on the Drug Attitude Inventory-10 items (DAl-10) and the Kemp's 7-point scale at baseline, 6-month, and 12-month follow-up.

the potential benefits of psychotropic medications, including LAIs. Unfortunately, real-world psychiatric services often lack sufficient resources - such as availability of time and personnelto adequately inform and support the needs of people suffering from severe mental illnesses. For instance, a study by Potkin et al. (58) reported that clinicians spent an average time of 12 min with patients in treatment with LAIs, and that only $2 \%$ of this time was occupied by discussions about the adherence to medication. However, shared-decision making has been extensively suggested as a winning strategy to overcome barriers hampering the acceptance of LAIs by patients and improve the outcome (37).

The main limitation of the present study is that data were drawn from a naturalistic, observational study about the use 
TABLE 3 | Linear mixed effect models to estimate whether attitude toward treatment and treatment adherence predict symptoms changes over time.

\begin{tabular}{lccc}
\hline BPRS & \multicolumn{3}{c}{ Linear mixed effect models } \\
\cline { 2 - 4 } & $\boldsymbol{B}$ & $\mathbf{9 5 \%} \mathbf{~ C l}$ & $\boldsymbol{P}$ \\
\hline DAl-10 × (6 months vs. baseline $)$ & -0.03 & $-0.29,0.23$ & 0.81 \\
DAl-10 $\times(12$ months vs. baseline $)$ & -0.17 & $-0.44,0.10$ & 0.23 \\
Kemp's $\times(6$ months vs. baseline $)$ & 0.48 & $-0.43,1.39$ & 0.30 \\
Kemp's $\times(12$ months vs. baseline $)$ & 0.35 & $-0.57,1.26$ & 0.46 \\
\hline
\end{tabular}

Each model includes: BPRS as dependent variable, fixed effects: predictor (DAl-10 or Kemp's scale), time, predictor $\times$ time, age, and sex: random-effects: center, participant. BPRS, Brief Psychiatric Rating Scale; DAI-10, Drug Attitude Inventory; Kemp's, Kemp's 7-point scale.

TABLE 4 | Linear mixed effect models to estimate whether attitude toward treatment and treatment adherence predict symptoms changes over time, with sample stratified according to baseline severity (BPRS $<41$ or BPRS $\geq 41$ ).

\begin{tabular}{lccc}
\hline & $\boldsymbol{B}$ & $\mathbf{9 5 \%} \mathbf{C l}$ & $\boldsymbol{P}$ \\
\hline Baseline BPRS < $\mathbf{4 1}(\boldsymbol{n}=\mathbf{1 4 4})$ & & & \\
DAl-10 × (6 months vs. baseline) & -0.05 & $-0.35,0.24$ & 0.74 \\
DAl-10 $\times(12$ months vs. baseline $)$ & -0.35 & $-0.66,-0.05$ & 0.02 \\
Kemp's $\times(6$ months vs. baseline $)$ & -0.48 & $-1.51,0.55$ & 0.36 \\
Kemp's $\times(12$ months vs. baseline $)$ & -1.82 & $-2.88,-0.77$ & 0.001 \\
Baseline BPRS $\geq \mathbf{4 1}$ ( $\boldsymbol{n}=\mathbf{3 1 7})$ & & & \\
DAl-10 $\times(6$ months vs. baseline $)$ & -0.32 & $-0.61,-0.03$ & 0.03 \\
DAl-10 $\times(12$ months vs. baseline $)$ & -0.41 & $-0.71,-0.11$ & 0.007 \\
Kemp's $\times(6$ months vs. baseline $)$ & -1.48 & $-2.53,-0.43$ & 0.006 \\
Kemp's $\times(12$ months vs. baseline $)$ & -1.46 & $-2.51,-0.41$ & 0.006 \\
\hline
\end{tabular}

Each model includes: BPRS as dependent variable, fixed effects: predictor (DAl-10 or Kemp's scale), time, predictor $\times$ time, age, and sex: random-effects: center, participant. BPRS, Brief Psychiatric Rating Scale; DAI-10, Drug Attitude Inventory; Kem's, Kemp's 7-point scale.

of LAIs in real-word clinical practice. Therefore, our results cannot be generalized to patients taking oral medications only, a situation in which patients' attitude and adherence to medication may play a more relevant role in symptoms changes. Moreover, our analyses did not take into account some important factors, such as the type of psychiatric diagnosis, the type of oral medication taken by participants, the duration of untreated illness, and the number of previous oral medication. However, a stratification according to these factors may have substantially reduced the sample size and thus the validity of our findings. Besides attitude toward treatment and adherence, future research should adopt tools evaluating the quality of patient-clinician relationship as well as insight levels to investigate more indepth the complex associations between these factors and clinical response in patients with severe mental illnesses.

In conclusion, the present study did not confirm our initial hypothesis that attitude toward medication and treatment adherence may represent potential predictors of changes in psychiatric symptoms over time in a naturalistic sample. However, severity represents a key factor, as patients with the most severe symptoms may benefit more precociously from LAI treatment due to improvement in attitude toward medication and treatment adherence. Our findings remark that it is fundamental to build and maintain a meaningful and reciprocal therapeutic alliance with patients suffering from severe mental illnesses over time. Such a relationship should involve a clear and comprehensive explanation of both the efficacy and the potential side effects of psychotropic drugs, including LAI, to convey greater awareness to the patients (38). A focus on patients' experience and preferences could help improve attitudes toward and appropriate use of LAI antipsychotics $(29,59)$. The increase of resources allocated to mental health services should be considered a priority to improve the long-term outcomes for psychiatric patients thus reducing the economic burden of chronic mental illnesses.

\section{DATA AVAILABILITY STATEMENT}

The raw data supporting the conclusions of this article will be made available by the authors, without undue reservation.

\section{ETHICS STATEMENT}

The study protocol was approved by the local ethics committee of the coordinating centre (Ethics Committee for Clinical Trials of the Provinces of Verona and Rovigo, protocol n.57622 of the 09/12/2015) and of each participating centre. The participants provided their written informed consent to participate in this study.

\section{AUTHOR CONTRIBUTIONS}

AAg and LF-P: statistical analyses and writing original draft. VP, AAm, and CC: data collection and revision of data literature. GM, GC, FB, and AD'A: supervision data collection, review and editing of the original draft, and scientific advisor of the project. GS, EA, and MA: review and editing of the original draft. CB and GO: writing protocol, designed the study, and scientific advisor of the project. All authors approved of the final draft of the manuscript before submission.

\section{FUNDING}

This work was supported by the Youth Foundation, Hong Kong (YF2012B-HX01).

\section{ACKNOWLEDGMENTS}

The STAR Network Investigators are: Corrado Barbui (Verona), Michela Nose' (Verona), Marianna Purgato (Verona), Giulia Turrini (Verona), Giovanni Ostuzzi (Verona), Maria Angela Mazzi (Verona), Davide Papola (Verona), Chiara Gastaldon (Verona), Samira Terlizzi (Verona), Federico Bertolini (Verona), Alberto Piccoli (Verona), Mirella Ruggeri (Verona), Pasquale De Fazio (Catanzaro), Fabio Magliocco (Catanzaro), Mariarita Caroleo (Catanzaro), Gaetano Raffaele (Catanzaro), Armando D’Agostino (Milano), Edoardo Giuseppe Ostinelli (Milano), Margherita Chirico (Milano), Simone Cavallotti (Milano), Claudio Lucii (Siena), Simone Bolognesi (Siena), Sara Debolini (Siena), Elisa Pierantozzi (Siena), Francesco Fargnoli (Siena), Maria Del Zanna (Siena), Alessandra Giannini (Siena), Livia 
Luccarelli (Siena), Alberto De Capua (Siena), Pasqua Maria Annese (Siena), Massimiliano Cerretini (Siena), Fiorella Tozzi (Siena), Nadia Magnani (Grosseto), Giuseppe Cardamone (Grosseto), Francesco Bardicchia (Grosseto), Edvige Facchi (Grosseto), Federica Soscia (Grosseto), Spyridon Zotos (Ferrara), Bruno Biancosino (Ferrara), Filippo Zonta (Treviso), Francesco Pompei (Treviso), Camilla Callegari (Varese), Daniele Zizolfi (Varese), Nicola Poloni (Varese), Marta Ielmini (Varese), Ivano Caselli (Varese), Edoardo Giana (Varese), Aldo Buzzi (Varese), Marcello Diurni (Varese), Anna Milano (Varese), Emanuele Sani (Varese), Roberta Calzolari (Varese), Paola Bortolaso (Varese Verbano), Marco Piccinelli (Varese Verbano), Sara Cazzamalli (Varese Verbano), Gabrio Alberini (Varese Verbano), Silvia Piantanida (Varese Verbano), Chiara Costantini (Varese Verbano), Chiara Paronelli (Varese Verbano), Angela Di Caro (Varese Verbano), Valentina Moretti (Reggio Emilia), Mauro Gozzi (Reggio Emilia), Chiara D’Ippolito (Reggio Emilia), Silva Veronica Barbanti (Reggio Emilia), Papalini Alessandro (Reggio Emilia), Mariangela Corbo (Chieti), Giovanni Martinotti (Chieti), Ornella Campese (Chieti), Federica Fiori (Chieti), Marco Lorusso (Chieti), Lucia Di Capro (Chieti), Daniela Viceconte (Chieti), Valerio Mancini (Chieti), Francesco Suraniti (Catania), Maria Salvina Signorelli (Catania), Eugenio Rossi (Bologna), Pasqualino Lupoli (Bologna), Marco Menchetti (Bologna), Laura Terzi (Bologna), Marianna Boso (Pavia), Paolo Risaro (Pavia), Giuseppe De Paoli (Pavia), Cristina Catania (Pavia), Ilaria Tarricone (Bologna), Valentina Caretto (Bologna), Viviana Storbini (Bologna), Roberta Emiliani (Bologna), Beatrice Balzarro (Bologna), Giuseppe Carrà (Monza), Francesco Bartoli (Monza), Tommaso Tabacchi (Monza), Roberto Nava (Monza), Adele Bono (Monza), Milena Provenzi (Monza), Giulia Brambilla (Monza), Flora Aspesi (Monza), Trotta (Monza),

\section{REFERENCES}

1. Morken G, Widen JH, Grawe RW. Non-adherence to antipsychotic medication, relapse and rehospitalisation in recent-onset schizophrenia. BMC Psychiatry. (2008) 8:32. doi: 10.1186/1471-244X-8-32

2. Emsley R, Nuamah I, Hough D, Gopal S. Treatment response after relapse in a placebo-controlled maintenance trial in schizophrenia. Schizophr Res. (2012) 138:29-34. doi: 10.1016/j.schres.2012.02.030

3. Emsley R, Chiliza B, Asmal L, Harvey BH. The nature of relapse in schizophrenia. BMC Psychiatry. (2013) 13:50. doi: 10.1186/1471-244X-13-50

4. Czobor P, Van Dorn RA, Citrome L, Kahn RS, Fleischhacker WW, Volavka J. Treatment adherence in schizophrenia: a patient-level meta-analysis of combined CATIE and EUFEST studies. Eur Neuropsychopharmacol. (2015) 25:1158-66. doi: 10.1016/j.euroneuro.2015.04.003

5. Takeuchi H, Siu C, Remington G, Fervaha G, Zipursky RB, Foussias G, et al. Does relapse contribute to treatment resistance? Antipsychotic response in first- vs. second-episode schizophrenia. Neuropsychopharmacology. (2019) 44:1036-42. doi: 10.1038/s41386-018-0278-3

6. Bowtell M, Ratheesh A, McGorry P, Killackey E, O’Donoghue B. Clinical and demographic predictors of continuing remission or relapse following discontinuation of antipsychotic medication after a first episode of psychosis. A systematic review. Schizophr Res. (2018) 197:9-18. doi: 10.1016/j.schres.2017.11.010

7. Andreasen NC, Liu D, Ziebell S, Vora A, Ho BC. Relapse duration, treatment intensity, and brain tissue loss in schizophrenia: a prospective longitudinal MRI study. Am J Psychiatry. (2013) 170:609-15. doi: 10.1176/appi.ajp.2013.12050674
Martina Tremolada (Monza), Gloria Castagna (Monza), Mattia Bava (Monza), Enrica Verrengia (Monza), Sara Lucchi (Monza), Maria Ginevra Oriani (Ancona), Michela Barchiesi (Ancona), Monica Pacetti (Forli), Andrea Aguglia (Genova), Laura Rosa Magni (Brescia), Giuseppe Rossi (Brescia), Rossella Beneduce (Brescia), Giovanni Battista Tura (Brescia), Laura Laffranchini (Brescia), Daniele Mastromo (Milano), Farida Ferrato (Milano), Francesco Restaino (Milano), Emiliano Monzani (Milano), Matteo Porcellana (Milano), Ivan Limosani (Milano), Lucio Ghio (Genova), Maurizio Ferro (Genova), Vincenzo Fricchione Parise (Avellino), Giovanni Balletta (Avellino), Lelio Addeo (Avellino), Elisa De Vivo (Avellino), Rossella Di Benedetto (Avellino), Federica Pinna (Cagliari), Bernardo Carpiniello (Cagliari), Mariangela Spano (Treviso), Marzio Giacomin (Treviso), Damiano Pecile (Mantova), Chiara Mattei (Fermo), Elisabetta Pascolo Fabrici (Trieste), Sofia Panarello (Trieste), Giulia Peresson (Trieste), Claudio Vitucci (Trieste), Tommaso Bonavigo (Trieste), Monica Pacetti (Forli'), Giovanni Perini (Verona), Filippo Boschello (Verona), Stefania Strizzolo (Vicenza), Francesco Gardellin (Vicenza), Massimo di Giannantonio (Chieti), Daniele Moretti (Savona), Carlo Fizzotti (Savona), Edoardo Cossetta (Savona), Luana Di Gregorio (Trento), Francesca Sozzi (Trento), Giancarlo Boncompagni (Bologna), Daniele La Barbera (Palermo), Giuseppe Colli (Palermo), Sabrina Laurenzi (Civitanova Marche), Carmela Calandra (Catania), Maria Luca (Catania).

\section{SUPPLEMENTARY MATERIAL}

The Supplementary Material for this article can be found online at: https://www.frontiersin.org/articles/10.3389/fpsyt. 2021.784366/full\#supplementary-material

8. Kane JM, Kishimoto T, Correll CU. Non-adherence to medication in patients with psychotic disorders: epidemiology, contributing factors and management strategies. World Psychiatry. (2013) 12:216-26. doi: 10.1002/wps.20060

9. Carbon M, Correll CU. Clinical predictors of therapeutic response to antipsychotics in schizophrenia. Dialogues Clin Neurosci. (2014) 16:50524. doi: 10.31887/DCNS.2014.16.4/mcarbon

10. García S, Martínez-Cengotitabengoa M, López-Zurbano S, Zorrilla I, López P, Vieta E, et al. Adherence to antipsychotic medication in bipolar disorder and schizophrenic patients: a systematic review. J Clin Psychopharmacol. (2016) 36:355-71. doi: 10.1097/JCP.0000000000000523

11. Kikkert MJ, Dekker J. Medication adherence decisions in patients with schizophrenia. Prim Care Companion CNS Disord. (2017) 19:17n02182. doi: 10.4088/PCC.17n02182

12. Velligan DI, Sajatovic M, Hatch A, Kramata P, Docherty JP. Why do psychiatric patients stop antipsychotic medication? A systematic review of reasons for nonadherence to medication in patients with serious mental illness. Patient Prefer Adherence. (2017) 11:449-68. doi: 10.2147/PPA.S124658

13. El Abdellati K, De Picker L, Morrens M. Antipsychotic treatment failure: a systematic review on risk factors and interventions for treatment adherence in psychosis. Front Neurosci. (2020) 14:531763. doi: 10.3389/fnins.2020.531763

14. Ostuzzi G, Bighelli I, So R, Furukawa TA, Barbui C. Does formulation matter? A systematic review and meta-analysis of oral versus long-acting antipsychotic studies. Schizophr Res. (2017) 183:10-21. doi: 10.1016/j.schres.2016.11.010

15. Ostuzzi G, Bertolini F, Tedeschi F, et al. Oral and long-acting antipsychotics for relapse prevention in schizophrenia-spectrum disorders: a network meta-analysis of 92 randomized trials including 22,645 participants. World Psychiatry. 2022. [Epub ahead of print]. 
16. Kishimoto T, Robenzadeh A, Leucht C, Leucht S, Watanabe K, Mimura M, et al. Long-acting injectable vs. oral antipsychotics for relapse prevention in schizophrenia: a meta-analysis of randomized trials. Schizophr Bull. (2014) 40:192-213. doi: 10.1093/schbul/sbs150

17. Taipale H, Mehtala J, Tanskanen A, Tiihonen J. Comparative effectiveness of antipsychotic drugs for rehospitalization in schizophrenia-a nationwide study with 20-year follow-up. Schizophr Bull. (2018) 44:1381-7. doi: 10.1093/schbul/sbx176

18. Kishimoto T, Hagi K, Kurokawa S, Kane JM, Correll CU. Long-acting injectable versus oral antipsychotics for the maintenance treatment of schizophrenia: a systematic review and comparative meta-analysis of randomised, cohort, and pre-post studies. Lancet Psychiatry. (2021) 8:387404. doi: 10.1016/S2215-0366(21)00039-0

19. Haddad PM, Kishimoto T, Correll CU, Kane JM. Ambiguous findings concerning potential advantages of depot antipsychotics: in search of clinical relevance. Curr Opin Psychiatry. (2015) 28:216-21. doi: 10.1097/YCO.0000000000000160

20. Ostuzzi G, Bertolini F, Del Giovane C, Tedeschi F, Bovo C, Gastaldon C, et al. Maintenance treatment with long-acting injectable antipsychotics for people with nonaffective psychoses: a network meta-analysis. Am J Psychiatry. (2021) 178:424-36. doi: 10.1176/appi.ajp.2020.20071120

21. Nasrallah HA. Triple advantages of injectable long acting second generation antipsychotics: relapse prevention, neuroprotection, and lower mortality. Schizophr Res. (2018) 197:69-70. doi: 10.1016/j.schres.2018.02.004

22. Chaudhary K, Patel MM, Mehta PJ. Long-acting injectables: current perspectives and future promise. Crit Rev Ther Drug Carrier Syst. (2019) 36:137-81. doi: 10.1615/CritRevTherDrugCarrierSyst.2018025649

23. de Filippis R, De Fazio P, Gaetano R, Steardo L, Cedro C, Bruno $\mathrm{A}$, et al. Current and emerging long-acting antipsychotics for the treatment of schizophrenia. Expert Opin Drug Safety. (2021) 20:77190. doi: 10.1080/14740338.2021.1910674

24. NICE. Psychosis and Schizophrenia in Adults: Prevention and Management. Clinical guideline [CG178] (2014). Available online at: https://www.nice.org. uk/guidance/cg178 (accessed September 1, 2020).

25. Galletly C, Castle D, Dark F, Humberstone V, Jablensky A, Killackey E, et al. Royal Australian and New Zealand College of Psychiatrists clinical practice guidelines for the management of schizophrenia and related disorders. Aust $N$ Z J Psychiatry. (2016) 50:410-72. doi: 10.1177/0004867416641195

26. Barnes TR, Drake R, Paton C, Cooper SJ, Deakin B, Ferrier IN, et al. Evidencebased guidelines for the pharmacological treatment of schizophrenia: updated recommendations from the British Association for Psychopharmacology. $J$ Psychopharmacol. (2020) 34:3-78. doi: 10.1177/0269881119889296

27. Geerts P, Martinez G, Schreiner A. Attitudes towards the administration of long-acting antipsychotics: a survey of physicians and nurses. BMC Psychiatry. (2013) 13:58. doi: 10.1186/1471-244X-13-58

28. Heres S, Lambert M, Vauth R. Treatment of early episode in patients with schizophrenia: the role of long-acting antipsychotics. Eur Psychiatry. (2014) 29(Suppl. 2):1409-13. doi: 10.1016/S0924-9338(14)70001-X

29. Patel MX, Bishara D, Jayakumar S, Zalewska K, Shiers D, Crawford MJ, et al. Quality of prescribing for schizophrenia: evidence from a national audit in England and Wales. Eur Neuropsychopharmacol. (2014) 24:499509. doi: 10.1016/j.euroneuro.2014.01.014

30. Stevens GL, Dawson G, Zummo J. Clinical benefits and impact of early use of long-acting injectable antipsychotics for schizophrenia. Early Interv Psychiatry. (2016) 10:365-77. doi: 10.1111/eip.12278

31. Grover S, Sahoo S, Subodh BN, Malhotra N, Dua D, Avasthi A. Attitude and perceptions of patients towards long-acting depot injections (LAIs). Asian J Psychiatr. (2019) 44:200-8. doi: 10.1016/j.ajp.2019.07.052

32. Correll CU, Lauriello J. Using long-acting injectable antipsychotics to enhance the potential for recovery in schizophrenia. J Clin Psychiatry. (2020) 81:MS19053AH5C. doi: 10.4088/JCP.MS19053AH5C

33. Jaeger M, Rossler W. Attitudes towards long-acting depot antipsychotics: a survey of patients, relatives and psychiatrists. Psychiatry Res. (2010) 175:5862. doi: 10.1016/j.psychres.2008.11.003

34. Sajatovic M, Ross R, Legacy SN, Correll CU, Kane JM, Di Biasi F, et al. Identifying patients and clinical scenarios for use of long-acting injectable antipsychotics-expert consensus survey part 1. Neuropsychiatr Dis Treat. (2018) 14:1463-74. doi: 10.2147/NDT.S167394
35. Lindenmayer JP, Glick ID, Talreja H, Underriner M. Persistent barriers to the use of long-acting injectable antipsychotics for the treatment of Schizophrenia. J Clin Psychopharmacol. (2020) 40:346-9. doi: 10.1097/JCP.0000000000001225

36. Curto M, Fazio F, Ulivieri M, Navari S, Lionetto L, Baldessarini RJ. Improving adherence to pharmacological treatment for schizophrenia: a systematic assessment. Expert Opin Pharmacother. (2021) 22:114355. doi: 10.1080/14656566.2021.1882996

37. Coulter A. Shared decision making: everyone wants it, so why isn't it happening? World Psychiatry. (2017) 16:117-8. doi: 10.1002/wps.20407

38. Fiorillo A, Barlati S, Bellomo A, Corrivetti G, Nicolò G, Sampogna G, et al. The role of shared decision-making in improving adherence to pharmacological treatments in patients with schizophrenia: a clinical review. Ann Gen Psychiatry. (2020) 19:43. doi: 10.1186/s12991-020-00293-4

39. von Elm E, Altman DG, Egger M, Pocock SJ, Gøtzsche PC, Vandenbroucke JP. The Strengthening the Reporting of Observational Studies in Epidemiology (STROBE) statement: guidelines for reporting observational studies. J Clin Epidemiol. (2008) 61:344-9. doi: 10.1016/j.jclinepi.2007.11.008

40. Ostuzzi G, Mazzi MA, Terlizzi S, Bertolini F, Aguglia A, Bartoli F, et al. Factors associated with first-versus second-generation long-acting antipsychotics prescribed under ordinary clinical practice in Italy. PLoS ONE. (2018) 13:e0201371. doi: 10.1371/journal.pone.0201371

41. Bertolini F, Ostuzzi G, Pievani M, Aguglia A, Bartoli F, Bortolaso P, et al. The STAR Network Investigators. Comparing long-acting antipsychotic discontinuation rates under ordinary clinical circumstances: a survival analysis from an observational, pragmatic study. CNS Drugs. (2021) 35:65565. doi: 10.1007/s40263-021-00809-w

42. Barbui C, Bighelli I, Carrà G, Castellazzi M, Lucii C, Martinotti $\mathrm{G}$, et al. Antipsychotic dose mediates the association between polypharmacy and corrected QT interval. PLoS ONE. (2016) 11:e0148212. doi: 10.1371/journal.pone.0148212

43. Carrà G, Crocamo C, Bartoli F, Lax A, Tremolada M, Lucii C, et al. STAR Network Group. First-generation antipsychotics and QTc: any role for mediating variables? Hum Psychopharmacol. (2016) 31:3138. doi: 10.1002/hup. 2540

44. World Medical Association. World Medical Association Declaration of Helsinki: ethical principles for medical research involving human subjects. JAMA. (2013) 310:2191-4. doi: 10.1001/jama.2013.281053

45. Barbui C, Bertolini F, Bartoli F, Calandra C, Callegari C, Carrà $\mathrm{G}$, et al. STAR Network Investigators. Reasons for initiating longacting antipsychotics in psychiatric practice: findings from the STAR Network Depot Study. Ther Adv Psychopharmacol. (2020) 10:2045125320978102. doi: 10.1177/2045125320978102

46. Bartoli F, Ostuzzi G, Crocamo C, Corbo M, D’Agostino A, Martinotti G, et al. Clinical correlates of paliperidone palmitate and aripiprazole monohydrate prescription for subjects with schizophrenia-spectrum disorders: findings from the STAR Network Depot Study. Int Clin Psychopharmacol. (2020) 35:214-20. doi: 10.1097/YIC.0000000000000317

47. Roncone R, Tozzini C, Mazza M, de Risio A, Giosuè P, Morosini P, et al Validation of the Italian version of the self-report insight scale. Epidemiol Psichiatr Soc. (2003) 12:63-75. doi: 10.1017/S1121189X00006060

48. Leucht S, Kane JM, Kissling W, Hamann J, Etschel E, Engel R. Clinical implications of brief psychiatric rating scale scores. Br J Psychiatry. (2005) 187:366-71. doi: 10.1192/bjp.187.4.366

49. Roncone R, Ventura J, Impallomeni M, Falloon IR, Morosini PL, Chiaravalle E, et al. Reliability of an Italian standardized and expanded Brief Psychiatric Rating Scale (BPRS 40) in raters with high vs. low clinical experience. Acta Psychiatr Scand. (1999) 100:229-36. doi: 10.1111/j.1600-0447.1999.tb1 0850.x

50. Rossi A, Arduini L, De Cataldo S, Stratta P. Subjective response to neuroleptic medication: a validation study of the Italian version of the drug attitude inventory (DAI). Epidemiol Psichiatr Soc. (2001) 10:10714. doi: 10.1017/S1121189X00005182

51. Kemp R, Hayward P, Applewhaite G, Everitt B, David A. Compliance therapy in psychotic patients: randomised controlled trial. BMJ. (1996) 312:3459. doi: 10.1136/bmj.312.7027.345

52. Lian L, Kim DD, Procyshyn RM, Fredrikson DH, Cázares D, Honer WG, et al. Efficacy of long-acting injectable versus oral antipsychotic drugs in early 
psychosis: A systematic review and meta-analysis. Early Interv Psychiatry. (2021). [Epub ahead of print]. doi: 10.1111/eip.13202

53. Vohs JL, George S, Leonhardt BL, Lysaker PH. An integrative model of the impairments in insight in schizophrenia: emerging research on causal factors and treatments. Expert Rev Neurother. (2016) 16:1193204. doi: 10.1080/14737175.2016.1199275

54. Hogan TP, Awad AG. Subjective response to neuroleptics and outcome in schizophrenia: a re-examination comparing two measures. Psychol Med. (1992) 22:347-52. doi: 10.1017/S00332917000 30282

55. Karthik MS, Warikoo N, Chakrabarti S, Grover S, Kulhara P. Attitudes towards antipsychotics among patients with schizophrenia on first- or second-generation medications. Indian J Psychol Med. (2014) 36:28893. doi: $10.4103 / 0253-7176.135382$

56. De Berardis D, Marini S, Carano A, Lang AP, Cavuto M, Piersanti M, et al. Efficacy and safety of long-acting injectable atypical antipsychotics: a review. Curr Clin Pharmacol. (2013) 8:256-64. doi: 10.2174/157488471130899 90056

57. Bell M, Fiszdon J, Richardson R, Lysaker P, Bryson G. Are selfreports valid for schizophrenia patients with poor insight? Relationship of unawareness of illness to psychological self-report instruments. Psychiatry Res. (2007) 151:37-46. doi: 10.1016/j.psychres.2006. 04.012

58. Potkin S, Bera R, Zubek D, Lau G. Patient and prescriber perspectives on long-acting injectable (LAI) antipsychotics and analysis of in-office discussion regarding LAI treatment for schizophrenia. BMC Psychiatry. (2013) 13:261. doi: 10.1186/1471-244X-13-261
59. Patel MX, De Zoysa N, Bernadt M, David A. Depot and oral antipsychotics: patient preferences and attitudes are not the same thing. J Psychopharmacol. (2009) 23:789-96. doi: 10.1177/0269881108092124

Conflict of Interest: GM has been a consultant and/or a speaker and/or has received research grants from Angelini, Doc Generici, Janssen, Lundbeck, Otsuka, and Pfizer.

The remaining authors declare that the research was conducted in the absence of any commercial or financial relationships that could be construed as a potential conflict of interest.

Publisher's Note: All claims expressed in this article are solely those of the authors and do not necessarily represent those of their affiliated organizations, or those of the publisher, the editors and the reviewers. Any product that may be evaluated in this article, or claim that may be made by its manufacturer, is not guaranteed or endorsed by the publisher.

Copyright (C) 2021 Aguglia, Fusar-Poli, Amerio, Placenti, Concerto, Martinotti, Carrà, Bartoli, D'Agostino, Serafini, Amore, Aguglia, Ostuzzi, Barbui and the STAR Network Investigators. This is an open-access article distributed under the terms of the Creative Commons Attribution License (CC BY). The use, distribution or reproduction in other forums is permitted, provided the original author(s) and the copyright owner(s) are credited and that the original publication in this journal is cited, in accordance with accepted academic practice. No use, distribution or reproduction is permitted which does not comply with these terms. 